\title{
Is there a Low Risk of Multiple Sclerosis in HIV Patients?
} \author{
Martelo $^{3}$ and Marco Orsini*3 \\ ${ }^{1}$ National Institute of Traumatology and Orthopedics (INTO), Brazil \\ ${ }^{2}$ Fluminense Federal University (UFF), MMC Division of Neurology; Brazil \\ ${ }^{3}$ Severino Sombra University, Professional Master in Applied Health Sciences, Brazil
}

Celmir De Oliveira Vilaça ${ }^{1,2}$, Marco Antônio Araujo Leite ${ }^{2}$, Rossano Fiorelli³, Pietro Novelino ${ }^{3}$, Ricardo

Received: April 26, 2018; Published: May 04, 2018

*Corresponding author: Marco Orsini, Severino Sombra University, Professional Master in Applied Health Sciences. Vassouras, RJ, Brazil. Master Program in Science Rehabilitation-UNISUAM-RJ, Rua Tavares de Macedo, 95/902, CEP 24220-215, Icaraí, Niterói-RJ, Brazil

\begin{abstract}
Objectives: Discuss the hypothesis of HIV infection to reduce the risk of Multiple Sclerosis.

Method: Non-systematic review using a Google Scholar platform of articles with the keywords: "HIV" or "Human Immunodeficiency Virus" associated with the term "multiple sclerosis" in the title of the journal.

Results and Discussion: 44 articles were used in English language for this manuscript.

Conclusion: There are few cases of patients with HIV and multiple sclerosis. The HIV infection may decrease risks of multiple sclerosis. The study of this association may contribute to the understanding of the physiopathology in inflammatory diseases of the central nervous system and the use of drugs for HIV infection in multiple sclerosis.
\end{abstract}

Keywords: Multiple Sclerosis; HIV; Anti-Retroviral Agents

Abbreviations: MS: Multiple Sclerosis; CNS: Central Nervous System; EB: Epstein Barr Virus; VZ: Varicella Zoster Virus

\section{Introduction}

Multiple sclerosis (MS) is a chronic inflammatory disease of the central nervous system (CNS) of an autoimmune nature with lymphocyte involvement. Activated lymphocytes cross the bloodbrain barrier and cause demyelination and axonal loss [1,2]. The disease affects 2.5 million people worldwide. It has a predominance in females and a higher incidence in people between 20 and 40 years of age [2]. This distribution of age and sex is also observed in samples of the Brazilian population [3]. Multiple sclerosis (MS) is the leading non-traumatic incapacitating neurological disease in young people economically active [4]. It can be subdivided into 4 clinical subtypes: the most frequent remission form, secondarily progressive form, primarily progressive and benign form [5]. Immunopathological findings include the predominant activation of CD8+ T lymphocytes on other T and B lymphocyte subpopulations, including CD4+ lymphocytes. The diagnosis is essentially clinical with widespread signs and symptoms in space and time with imaging studies demonstrating the presence of white matter lesions [1]. On the other hand, HIV infection (Human Immunodeficiency Virus) affects about 34 million people worldwide [6]. The HIV virus is subdivided into types I and II. Type I was discovered in a French patient in the year 1983 and the type II in Africa in 1986 [7]. Data from 2010 estimated about 620 thousand people infected with HIV in Brazil. The infection predominates in the age range between 20 and 40 years in males and 30 to 50 years in females [8].

Both MS and HIV infection are examples of immune diseases where there is a change in the inflammatory response of CD4+ T and CD8+ T lymphocytes. In addition, we observed a higher incidence of these diseases in similar age groups. Despite this, there are few reported cases of patients affected by both diseases [9]. The risk of multiple sclerosis in HIV patients could be up to $62 \%$ lower when compared to the general population [2]. In addition, both diseases have opposite gender prevalence, with MS being more prevalent in women and HIV in men [10]. In addition, in sub-Saharan Africa where there is a higher rate of HIV infection, there is a lower risk of MS compared to the United States and Europe [11]. On the other hand, many of the reported cases of individuals with both diseases could represent a diagnostic error because they predate the 1990s. At that time, the current clinical, laboratory and imaging criteria 
for the diagnosis of MS were not used yet $[12,13]$. Research on cases involving both diseases may increase understanding about the functioning of the immune system in the various inflammatory diseases of the CNS.

\section{Method}

We did a non-systematic review of articles on the Google Scholar platform. We looked for articles in English whose title presented the association of the descriptors: "HIV" or "Human Immunodeficiency Virus" associated with the descriptor "multiple sclerosis" in the title of the journal. We obtained a total of 102 articles with this association. We have included trial articles, review articles, case reports, or expert opinions with no specific criteria to exclude them except English content. Some articles were chosen from the references obtained from the periodicals of the initial search. We have used a total of 44 articles in English to elaborate this one.

\section{Results and Discussion \\ Viral Hypothesis in Multiple Sclerosis}

The interface of environmental and genetic factors is recognized in the pathophysiology of Multiple Sclerosis [14,15]. Among the environmental factors there is the possibility of the participation of some viruses in the pathophysiology of MS. Epstein Barr virus (EB) is the most consistently associated with the occurrence of the disease [16]. EB is an oncogenic virus of the herpesvirus family discovered in the 1960s in cases of lymphoma. Ninety percent of the individuals acquired EB infection in the first decade of life [17]. The virus remains latent in $B$ lymphocytes since the initial infection. Thus, most patients with multiple sclerosis are infected by the EB virus years before the first outbreak. Epstein Barr is present in almost all cases of MS. Infected B lymphocytes would participate in the inflammatory responses responsible for MS outbreaks in the future. Unlike EB, the infection by most of the other viruses implicated in MS occurs approximately two weeks before the first outbreak [18]. There is also the hypothesis of the collaboration of some retroviruses known as HERV or Human Endogenous Retroviruses.

These may constitute up to $8 \%$ of the human genome and many of them have not yet been fully mapped through DNA analysis $[19,20]$. The proteins and primary transcripts of HERVs have often been detected in the blood and in the CNS in situations of neuforinflammation [21]. There are also researches on the involvement of varicella zoster virus (VZ) in the etiology of multiple sclerosis. VZ may act as a risk factor in areas where the frequency of multiple sclerosis is low [22]. VZ virus as well as EB is acquired in childhood or adolescence and may remain latent in the host for decades [23]. Variations in the amount of VZ DNA obtained in the CSF were demonstrated depending on the MS subtype. Larger quantities are seen during an outbreak and smaller during periods of remission. In primary progressive forms there can be continuous production at low scale [24]. Moreover, patients with MS present high antibody titres against viral particles of the most different types.

These particles could cause continuous activation of the immune system affecting immune pathways responsible for the onset of lesions typical of Multiple Sclerosis [14]. Besides, up to $30 \%$ of outbreaks of established MS may be associated with acute bacterial or viral infectious processes [22]. Viral infections are also implicated in other CNS demyelinating diseases such as Acute Disseminated Encephalomyelitis (ADEM). We should also highlight the myelopathy associated with HTLV I and II or tropical spastic paraparesis as another inflammatory CNS disease caused by a retrovirus [25]. This disease, although presenting more frequently with images indicative of lesion of the white matter of the spinal cord, may also present demyelinating encephalic lesions suggestive of MS in nuclear magnetic resonance (NMR) [26].

\section{HIV Infection and Demyelinating Diseases}

Patients with HIV infection present neurological complications in up to 40 to $70 \%$ of cases throughout their evolution [27]. Among these complications encephalopathy by HIV is mentioned, caused directly by viral action and diseases associated with immunosuppression such as neurotoxoplasmosis, progressive multifocal leukoencephalopathy (PML), cryptococcosis and primary CNS lymphoma [28]. The white matter lesions or also called demyelinating more frequent in HIV are the encephalopathy by HIV and the PML $[9,28,29]$. The HIV encephalopathy is clinically characterized by progressive cognitive decline associated with behavioraland motor symptoms [7]. Previously, HIV encephalopathy was observed only in cases of advanced immunosuppression with high viral load and low CD4+ lymphocyte counts and was manifested clinically through an advanced dementia. The advents of antiretroviral therapy (ART) decreased the number of severe cases but were replaced by several mild cognitive impairment conditions. Currently, in the pathophysiology of HIV encephalopathy, we also consider a possible CNS attack by the use of long-term ART $[7,30]$.

Another cause of white matter injury is PML. PML was initially described in patients with HIV but also occurs in association with other states of immunosuppression such as lymphomas, acute or chronic leukemias, chemotherapy or treatment with immunosuppressive drugs [31]. Just as HIV encephalopathy, PML most often occurs in advanced stages of HIV immunosuppression. The diagnosis of PML is associated with the presence of positive serology for the JC virus. The JC virus is of universal distribution and around $80 \%$ of the general population has antibodies against the virus. Primary infection is usually asymptomatic with virus survival in the renal parenchyma. Immunodepression states lead to reactivation of the virus and destruction of the oligondendrocytes responsible for the formation of the myelin sheath [32]. Sometimes the diagnosis of PML is difficult because the DNA of the virus is not detected in $100 \%$ of cases [14,33]. Some findings may help differentiate HIV encephalopathy and PML from multiple sclerosis outbreaks. Both HIV encephalopathy and PML do not improve with corticoid pulse therapy as it usually occurs during a MS outbreak $[14,33]$.

Another characteristic for differential diagnosis is neuroimaging findings. HIV encephalopathy and PML in MRI exams do not present the appearance of black holes typical of MS [29,34]. Also lesions of HIV encephalopathy do not usually present contrast uptake in MRI. In PML either there is no contrast uptake or it is small and usually 
at the periphery of the lesion [30,32]. When the neuropathological aspects are analyzed, the central characteristic of MS is the presence of perivascular demyelination. In progressive multifocal leukoencephalopathy there are inclusions in oligodendrocytes and the presence of bizarre astrocytes. HIV encephalopathy is characterized by the presence of multinucleated giant cells $[29,34]$. Another rare white matter disease in HIV patients is posterior reversible leukoencephalopathy syndrome (PRES). Corresponds to a vasogenic lesion preferentially observed in the posterior encephalon with white matter edema. PRES would originate from an endothelial vascular disorganization in HIV patients. This vascular alteration would be independent of CD4+ counts and the presence of classic PRES risk factors such as hypertension and eclampsia [35].

\section{Association Between HIV Infection and Multiple Sclerosis}

The first reports of patients presenting both MS and HIV infection go back to 1989 [29]. There are cases of HIV whose patients have improved lesions and decreased the number of MS outbreaks after starting antiretroviral treatment. In addition, most of them present a high CD 4 + count at the time of diagnosis of MS [9]. There are also cases of atypical MS or with findings suggestive of optic neuromyelitis (NMO), also known as Devic's disease or Devic's syndrome, with improvement after the introduction of ART [36]. In NMO, the presence of HIV works by affecting T cells and signaling for the proliferation of antibody-producing B lymphocytes. Such antibodies, among them anti-aquaporin 4, would be responsible for the lesions of the NMO [37]. Rarely, as in other viral infections, there is a suggestive appearance of ADEM in patients with HIV. In this case the MRI lesions are characterized by single-phase character through a single outbreak. ADEM in individuals with HIV most often appear during the period of seroconversion $[33,38]$.

Remission of acute disseminated encephalomyelitis lesions occurs in up to four weeks in most cases [39]. There are also demyelinating tumefying forms corresponding to an intermediary entity between ME and ADEM. These forms are characterized in the MRI by a single contrast-sensitive demyelinating lesion and with mass effect being confused with a tumor lesion [28] Some researchers believe there is a "multiple sclerosis-like syndrome" caused by the HIV virus with clinical manifestations indistinguishable from a MS outbreak. This syndrome would originate from recent HIV infection [14,27]. It would represent an analogue of Guillain-Barré syndrome or acute inflammatory demyelinating polyradiculopathy that might occur in the peripheral nervous system of individuals with HIV during the initial period of seroconversion $[29,34]$. In such cases there is the possibility of involved molecular mimicry. Thus, antibodies against the viral components could attack components of the myelin sheath. This hypothesis ratifies the discovery of the production of antibodies against the HIV lipoprotein gp120. This antibody reacts against three brain proteins, one of which is unique to the CNS [36].

The time interval between the manifestations of both diseases and the need for the use of ART for HIV treatment is another matter for debate. There are cases of decades between the emergence of
MS outbreaks and the diagnosis of HIV infection, suggesting no causal link or indicating random association. Other cases are at different stages of HIV infection with greater or lesser need for ART. Finally there are cases with an evident proximity of time between HIV infection and outbreaks increasing the possibility of association [14]. HIV infection could prevent the occurrence of autoimmune lesions typical of MS by modifying the immune system response. The infection would affect the functioning of CD4+ / CD8+ lymphocytes and MS-related signaling pathways [12]. Patients with MS and HIV undergoing treatment have similar numbers of CD4+ lymphocytes. Patients not treated for HIV have lower CD4+ lymphocytes than patients with MS. Decreased CD4+ lymphocytes could explain the protection of HIV patients at the onset of MS [10]. Decreased CD4+ lymphocytes also affect the activation of T lymphocytes (treg). These lymphocytes participate in pathways responsible CNS injury in multiple sclerosis [12].

On the other hand, it is possible that antiretroviral therapy is responsible for reducing the risk of MS in those infected by HIV. In this case ART could affect other retroviruses postulated in the pathophysiology of MS. Currently there are MS investigations involving the experimental use of antiretrovirals such as raltegravir and the monoclonal antibody GNbAC1 used against HERVs [2]. Raltegravir is an antiretroviral acting on the integrase enzyme. Inhibition of the integrase would not allow fusion of the virus DNA into the nuclear DNA of the host cell [36]. However, there is no indication for the use of antiretrovirals to combat HIV infection in multiple sclerosis. Meanwhile, considering the antiretrovirals already available in the market tenofovir is a drug whose chemical structure has a fumaric ring. Fumarate is the basis of the difumarate formulation. The difumarate is a drug used in MS reducing up to $50 \%$ the occurrence of outbreaks. It has immunomodulatory action by inhibiting inflammatory cytokines such as TNF- $\alpha$ and interleukins $1-\beta$ and IL- 6 by glial cells $[1,12,38]$.

Individuals at different stages of HIV infection have different needs regarding the use of antiretroviral therapy. There is no estimate of the time needed to use ART in HIV patients until the drugs have a protective effect on the development of MS or a decrease in the number of outbreaks in already established cases.40 In addition to the development of new drugs, there have been changes in the posture regarding HIV treatment with ART over the last two decades. The recommendations now indicate more aggressive treatment in the early stages of infection, or treatment only in more advanced stages. Such discussion is due to the effects of ART. Treatment also presents changes dependent on viral load and CD4+ lymphocyte counts $[19,40]$. The decrease in viral load and specially the recovery of CD4+ lymphocyte values when initiating ART in HIV patients is another issue for debate. Some of these individuals present clinical and radiological worsening of CNS in MRI images suggesting an inflammatory immune reconstitution syndrome (IRIS).

In such cases it may be difficult to distinguish a MS outbreak [9]. Prospective studies indicate the occurrence of IRIS by ART in up to $40 \%$ of cases [33]. IRIS in addition to occurring in HIV patients when initiating ART may also occur in patients with 
MS after immunosuppressive withdrawal [9]. In the case of immunosuppressant's, the suspension of natalizumab or its withdrawal through plasmapheresis may lead to an IRIS associated to PML. This risk attributed to natalizumab occurs in both MS and Chron's disease [41]. IRIS becomes unlikely when there is no state of immunosuppression at the time of the introduction of ART or there is great time interval between immunosuppressive therapy changes and worsening of clinical and image symptoms [29]. An HIV infected patient may present IRIS by the introduction of ART up to two years after initiation of therapy [37]. The laboratory evaluation of the cases of patients with HIV and MS evidenced moderately high protein levels in the cerebrospinal fluid and absence of oligoclonal bands in most cases [27]. There is also elevation of myelin basic protein levels to CSF. Elevated myelin basic protein levels in the CSF are most commonly found in multiple sclerosis when compared to HIV neuroinfection in the CNS [36].

Some authors contest the low risk of HIV in patients with MS. They proposed that chronic HIV infection leads to chronic activation of the immune system with increased risk of multiple sclerosis as with other retroviruses [11]. Patients with HIV and elevation of CD4+ with low viral load present activation of the Th17 lymphocyte subpopulation. Th17 lymphocytes have a central role in autoimmune processes in the CNS, including MS outbreaks $[36,42]$. Finally, we found only one report of a Brazilian patient with an association between HIV and MS. He presented a fulminating form of multiple sclerosis. This report dates back to 1991, long before the new criteria for MS and current antiretroviral therapy [43]. There is also a case of a Brazilian woman suffering from both diseases residing in Switzerland [44]. The small number of national cases reinforces the possibility of diagnostic errors or underreporting. The presence of underreporting could be higher in developing countries than rich countries [11].

\section{Conclusion}

All things considered, despite the high incidence of both diseases and similar age distribution, there are few cases of patients with HIV and multiple sclerosis associated. There is a hypothesis that HIV infection is a protective factor for the occurrence of multiple sclerosis. This protection can be attributed to both the presence of the HIV virus and antiretroviral therapy. The study of this association is important to understand the immunological mechanisms of inflammatory diseases of the CNS as well as the possibility of using therapies used in HIV infection in multiple sclerosis.

\section{References}

1. Huang WJ, Chen WW, Zhang X (2017) Multiple sclerosis: Pathology, diagnosis and treatments. Exp Ther Med 13(6): 3163-3166.

2. Insha Zahoor MK (2015) Human Immunodeficiency Virus and Multiple Sclerosis Risk: Probing for a Connection. J Mult Scler p. 2-5.

3. Vasconcelos CCF, Thuler LCS, Rodrigues BC, Calmon AB, Alvarenga RMP (2016) Multiple sclerosis in Brazil: A systematic review. Clin Neurol Neurosurg 151: 24-30.

4. Eskandarieh S, Heydarpour P, Minagar A, Pourmand S, Sahraian MA (2016) Multiple Sclerosis Epidemiology in East Asia, South East Asia and South Asia: A Systematic Review. Neuroepidemiology 46: 209-221.
5. Hurwitz BJ (2009) The diagnosis of multiple sclerosis and the clinical subtypes. Ann Indian Acad Neurol 12(4): 226-230.

6. Cornett JK, Kirn TJ (2013) Laboratory diagnosis of HIV in adults: A review of current methods. Clin Infect Dis 57(5): 712-718.

7. Miura Y, Koyanagi Y (2005) HIV encephalopathy. Rinshō Shinkeigaku Clin Neurol 45: 887-889.

8. De Souza SMB, Teles SA, Rezza G, Pezzotti P, Gir E (2013) Epidemiology of HIV infection in central Brazil: Data from voluntary counseling and testing centers. J Assoc Nurses AIDS Care 24: 503-511.

9. Anand P, Saylor D (2018) Multiple sclerosis and HIV: a case of multiple sclerosis-immune reconstitution inflammatory syndrome associated with antiretroviral therapy initiation. Int J STD AIDS.

10. Koudriavtseva T, Plantone D, Mandoj C, Giannarelli D, Latini A, et al. (2017) HIV and decreased risk of multiple sclerosis: role of low CD4+ lymphocyte count and male prevalence. J Neurovirol 23(1): 147-151.

11. Chin JH (2015) Multiple sclerosis and HIV-1 infection: case report of a HIV controller. J Neurovirol 21(4): 464-467.

12. Skarlis C, Gontika M, Katsavos S, Velonakis G, Toulas P, et al. (2017) Multiple sclerosis and subsequent human immunodeficiency virus infection: A case with the rare comorbidity, focus on novel treatment issues and review of the literature. In Vivo 31(5): 1041-1046.

13. Schippling S (2017) MRI for multiple sclerosis diagnosis and prognosis. Neurodegener Dis Manag 7: 27-29.

14. Facchini SA, Harding SA, Waldron RL (2002) Human immunodeficiency virus-1 infection and multiple sclerosis-like illness in a child. Pediatr Neurol 26: 231-235.

15. Haq E, Zahoor I, Khan M (2016) Association between Multiple Sclerosis Risk and Human Immunodeficiency Virus Infection: Insights and Challenges. In: Trending Topics in Multiple Sclerosis. InTech.

16. Dolei A (2018) The aliens inside us: HERV-W endogenous retroviruses and multiple sclerosis. Mult Scler J 24: 42-47.

17. Ascherio A, Munger KL (2010) Epstein-Barr Virus Infection and Multiple Sclerosis: A Review. J Neuroimmune Pharmacol 5(3): 271-277.

18. Pender MP (2011) The Essential Role of Epstein-Barr Virus in the Pathogenesis of Multiple Sclerosis. The Neuroscientist 17(4): 351-367.

19. Gold J, Goldacre R, Maruszak H, Giovannoni G, Yeates D, et al. (2015) HIV and lower risk of multiple sclerosis: beginning to unravel a mystery using a record-linked database study. J Neurol Neurosurg Psychiatry 86(1): 9-12.

20. Arru G, Mameli G, Astone V, Serra C, Huang YM, et al. (2007) Multiple Sclerosis and HERV-W/MSRV: A Multicentric Study. Int J Biomed Sci 3(4): 292-297.

21. Laska MJ, Brudek T, Nissen KK, Christensen T, Larsen AM, et al. (2011) Pathogenesis of multiple sclerosis: expression of HERV-Fc1: a human endogenous retrovirus. Retrovirology 8(2): 23.

22. Corona T, Flores J (2011) Herpes Zoster and Multiple Sclerosis. J Infect Dis 204: $177-178$.

23. Sotelo J, Corona T (2011) Varicella Zoster Virus and Relapsing Remitting Multiple Sclerosis. Mult Scler Int p. 1-5.

24. Ordoñez G, Martinez-Palomo A, Corona T, Pineda B, Flores-Rivera J, et al. (2010) Varicella zoster virus in progressive forms of multiple sclerosis. Clin Neurol Neurosurg 112(8): 653-657.

25. Bangham CRM, Araujo A, Yamano Y, Taylor GP (2015) HTLV-1-associated myelopathy/tropical spastic paraparesis. Nat Rev Dis Primer 1: 15012.

26. Morgan DJ, Caskey MF, Abbehusen C, Oliveira-Filho J, Araujo C, et al. (2007) Brain Magnetic Resonance Imaging White Matter Lesions Are Frequent in HTLV-I Carriers and Do Not Discriminate from HAM/TSP. AIDS Res Hum Retroviruses 23: 1499-1504. 
27. Coban A, Akman-Demir G, Ozsut H, Eraksoy M (2007) Multiple Sclerosis???Like Clinical and Magnetic Resonance Imaging Findings in Human Immunodeficiency Virus Positive-Case. The Neurologist 13: 154157.

28. Uriel A, Stow R, Johnson L, Varma A, du Plessis D, et al. (2010) Tumefactive Demyelination-An Unusual Neurological Presentation of HIV. Clin Infect Dis 51(10): 1217-1220.

29. González-Duarte A, Ramirez C, Pinales R, Sierra-Madero J (2011) Multiple sclerosis typical clinical and MRI findings in a patient with HIV infection. J Neurovirol 17: 504-508.

30. Loredana C, Petrescu AM, Moroti Constantinescu R (2012) HIV Encephalopathy-Now and Then In: Miscellanea on Encephalopathies. InTech.

31. Blessing MM, Raghunathan A, Erickson LA (2018) Progressive Multifocal Leukoencephalopathy. Mayo Clin Proc 93: 123-125.

32. Zucker BE, Stacpoole SRL (2018) Progressive multifocal leukoencephalopathy in the absence of immunosuppression. J Neurovirol 24(1): 119-122.

33. van Toorn R, Kritzinger F, Rabie H (2005) Acute demyelinating encephalomyelitis (ADEM), cryptococcal reactivation and disseminated Herpes simplex in an HIV infected child following HAART. Eur J Paediatr Neurol 9(5): 355-359.

34. Durán E, Gálvez J, Patrignani G, Izquierdo G (2004) Multiple sclerosislike illness in a HIV-1 patient. J Neurol 251(9): 1142-1144.

35. Birner B, Hirzel C, Wagner F, Waldegg G (2018) Posterior reversible encephalopathy syndrome in an HIV-infected patient on antiretroviral treatment: what is the risk factor? BMJ Case Rep.
36. Delgado SR, Maldonado J, Rammohan KW (2014) CNS demyelinating disorder with mixed features of neuromyelitis optica and multiple sclerosis in HIV-1 infection. Case report and literature review. J Neurovirol 20(5): 531-537.

37. Salazar R, Cerghet M, Shad A, Markowitz NP (2013) NMO-IgG positive relapsing longitudinally extensive transverse myelitis (LETM) in a seropositive HIV patient. Clin Neurol Neurosurg 115(9): 1873-1875.

38. Chalkley J, Berger JR (2014) Multiple sclerosis remission following antiretroviral therapy in an HIV-infected man. J Neurovirol 20: 640-643.

39. Naidoo A, Paruk H, Bhagwan B, Moodley A (2017) Atypical presentations of acute disseminated encephalomyelitis (ADEM) in HIV infection. J Neurovirol 23: 160-170.

40. Kop ML van der (2015) Does antiretroviral therapy for HIV reduce the risk of developing multiple sclerosis? J Neurol Neurosurg Psychiatry 86(1): 3 .

41. Kleinschmidt-Demasters BK, Miravalle A, Schowinsky J, Corboy J, Vollmer T (2012) Update on PML and PML-IRIS occurring in multiple sclerosis patients treated with natalizumab. J Neuropathol Exp Neurol 71(7): 604-617.

42. Roe C (2016) HIV immunodynamics and multiple sclerosis. J Neurovirol 22(2): 254-255.

43. Gray F, Chimelli L, Mohr M, Clavelou P, Scaravilli F, et al. (1991) Fulminating multiple sclerosis-like leukoencephalopathy revealing human immunodeficiency virus infection. Neurology 41(1): 105-109.

44. Graber P, Rosenmund A, Probst A, Zimmerli W (2000) Multiple sclerosislike illness in early HIV infection. AIDS 14(15): 2411-2413.

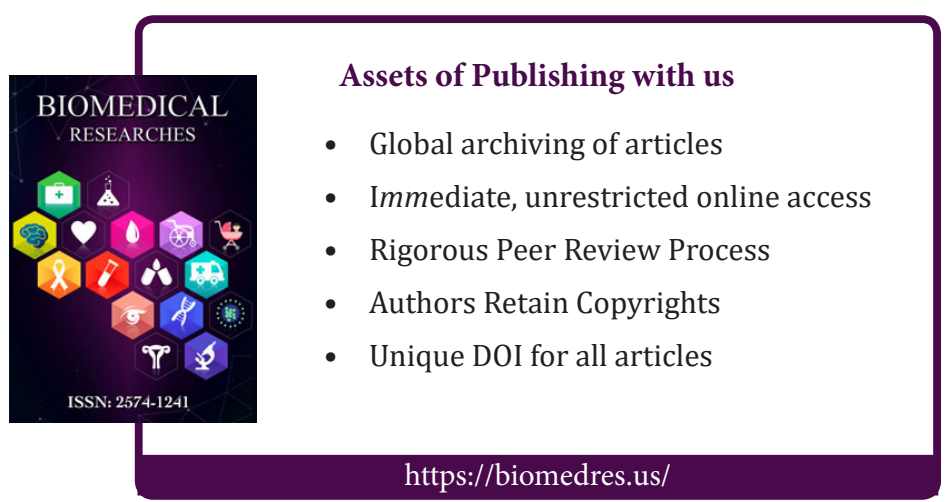

